\title{
A power law for the duration of high-flow states and its interpretation from a heterogeneous traffic flow perspective
}

\author{
Journal Article \\ Author(s): \\ Helbing, Dirk; Tilch, B. \\ Publication date: \\ 2009 \\ Permanent link: \\ https://doi.org/10.3929/ethz-b-000157281 \\ Rights / license: \\ In Copyright - Non-Commercial Use Permitted \\ Originally published in: \\ The European Physical Journal B 68(4), https://doi.org/10.1140/epjb/e2009-00092-8
}




\title{
A power law for the duration of high-flow states and its interpretation from a heterogeneous traffic flow perspective
}

\author{
D. Helbing ${ }^{1, a}$ and B. Tilch ${ }^{2}$ \\ 1 ETH Zurich, UNO D11, Universitätstr. 41, 8092 Zurich, Switzerland \\ ${ }^{2}$ II. Institut für Theoretische Physik, Pfaffenwaldring 57, 70550 Stuttgart, Germany
}

Received 19 June 2008 / Received in final form 17 December 2008

Published online 14 March 2009 - (c) EDP Sciences, Società Italiana di Fisica, Springer-Verlag 2009

\begin{abstract}
We study the duration of "high-flow states" in freeway traffic, defined as the time periods for which traffic flows exceed a given flow threshold. Our empirical data are surprisingly well represented by a power law. Moreover, the power law exponent for a two-lane freeway seems to be independent of the chosen flow threshold. In order to interpret this discovery, we investigate a simple theoretical model of heterogeneous traffic with overtaking maneuvers, which is able to reproduce both, the empirical power law and its exponent.
\end{abstract}

PACS. 89.40.Bb Land transportation - 89.75.Kd Patterns - 51.10.+y Kinetic and transport theory of gases

\section{Introduction}

Efficient transport systems are needed to fulfil the requirements of industrialized societies. However, studies of traffic physicists have shown that the capacity of a freeway is reduced by the breakdown of traffic flows [1-4], a phenomenon which is widely known as "capacity drop". Moreover, when the vehicle density increases, the traffic flow becomes metastable, i.e. a breakdown of traffic flow can be triggered by perturbations, if they exceed a critical perturbation threshold $[1,3,5,6]$. At even higher densities, traffic flow becomes linearly unstable, and a breakdown may be triggered by the slightest perturbation $[3,7]$.

Due to the dependence of traffic breakdowns on perturbations of the traffic flow, it is essential to know the characteristic properties of vehicle flows. While much attention has been paid to the measurement and explanation of the wide scattering of vehicle flows after the breakdown of free traffic (see e.g. Refs. [8-11] and citations therein), the features of flows before the breakdown have not found the attention they deserve. For example, the time period immediately before the breakdown is characterized by "high-flow states" [12-15] (see Fig. 1). These high-flow states are produced by small time gaps between subsequent vehicles, i.e. by vehicle clusters. Before we study these states, let us therefore shortly discuss some previous literature on vehicle platoons (see Ref. [3]).

\footnotetext{
a e-mail: dhelbing@ethz.ch
}

The formation of platoons is typically a result of the fact that vehicles do not behave identically. Driver-vehicle behavior is rather heterogeneous, which is typically reflected by fluctuation terms and distributed model parameters. The simplest models of stochastic and heterogeneous transport are particle hopping models with quenched disorder. For example, Evans [17], Krug and Ferrari [18], Karimipour [19,20], as well as Seppäläinen and Krug [21] study a simplified version of a model by Benjamini et al. [22]. It corresponds to the onedimensional driven lattice gas known as TASEP, but with particle-specific, constant jump rates $q_{\alpha}$. If overtaking is not allowed, Krug and Ferrari [18] find a sharp phase transition between a low-density regime, where all particles are queueing behind the slowest particle, and a high-density regime, where the particles are equally distributed. Note that the slow particles "feel free traffic" until the critical density is reached, at which traffic flow becomes unstable (cf. the truck curve in Fig. 2).

Close to the critical density, where the traffic flow becomes unstable, the growth of vehicle platoons is characterized by a power-law coarsening. If particles move ballistically with individual velocities $v_{\alpha}$ and form a platoon when a faster particle reaches a slower one, the platoon size $n_{\mathrm{pl}}(t)$ grows according to

$$
n_{\mathrm{pl}}(t) \sim t^{(\delta+1) /(\delta+2)},
$$

where the exponent $\delta$ characterizes the distribution $P_{0}(v) \sim\left(v-v_{\min }\right)^{\delta}$ of free velocities in the neighborhood 


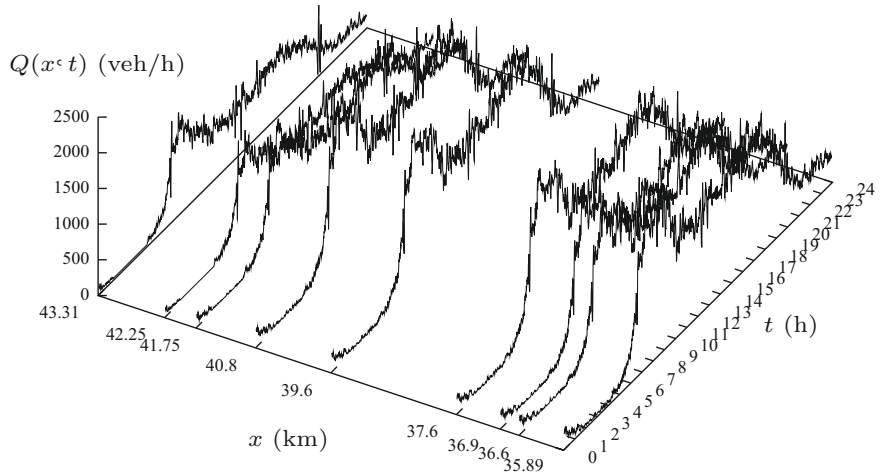

Fig. 1. Time-dependent traffic flows $Q(x, t)$ measured at different cross sections $x$ of a the Dutch freeway A9 on October 14, $1994^{1}$. Vehicles move from left to right. One can clearly see the large variability of high flows. Complementary empirical analyses show that the truck fraction on the freeway changes considerably with time as well, between about 8 and $80 \%$ trucks [9].

of the minimal desired velocity $v_{\min }[24-30]$. Beyond it, the differences among fast and slow particles become irrelevant, because there is so little space that all particles have to move slower than preferred.

Platoon formation and power-law coarsening has also been found in microscopic models with parallel update [24-27,29-32]. An example is the Nagel-Schreckenberg model with vehicle-specific slowdown probabilities [33,34].

An empirical measurement of platoon sizes has been performed by Neubert et al., based on velocity correlations between subsequent cars [35]. Our own approach will rather be focused on time periods with small time gaps between vehicles. For a model for platoon size distributions see Islam and Consul [36]. Furthermore, we point to the nice analyses of vehicle clustering based on master and Fokker-Planck equations [37-39], and to the determination of gap distributions for free traffic with vehicle platoons [40].

In the following, we will relate the distribution of platoon sizes with their growth dynamics, and study the importance of overtaking maneuvers for both. Before we start our theoretical considerations, however, Section 2 will present data of "high-flow states" and discuss their unexpected power-law statistics. Afterwards, Section 3 will present a theoretical explanation, based on platoon

\footnotetext{
${ }^{1}$ For reasons of illustration, the figure displays five-minute averages, so that the curves belonging to different cross sections of the freeway can be well enough resolved. (Two-minute data would show a larger variability. The variability is expected to be inversely proportional to the square root of the number of vehicles averaged over, i.e. to the square root of the sampling time.) For the analysis of the high-flow states, the sampling time should be chosen large enough to smoothen over statistical variations of the flow, but small enough to keep systematic features of the traffic dynamics. Two-minute averages, which are analyzed in the following, have been determined as an ideal compromise [41]. They also provide a sufficient number of data points for the analysis of high-flow states.
}
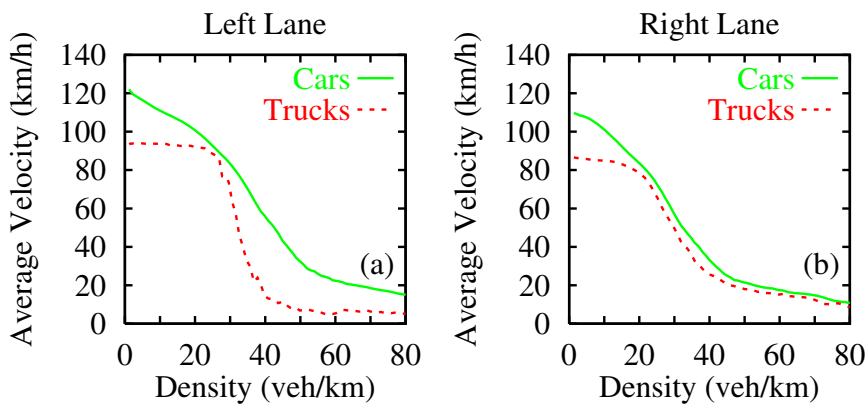

Fig. 2. (Color online) speed of vehicles as a function of the vehicle density in (a) the left lane and (b) the right (fast) lane (after [23]). The curves have been determined from single vehicle data of the Dutch freeway A9 close to Amsterdam. Note that the average velocity of trucks stays constant up to the critical density, where the speed of cars and trucks drops simultaneously. This incidates that trucks "feel" free traffic conditions at all vehicle densities upto the occurence of the transition to congested traffic.

formation due to overtaking maneuvers by slow vehicles, specifically trucks. This does, of course, not exclude the theoretical possibility of other explanations, e.g. based on the vehicle dynamics in single lanes. This alternative approach is discussed in Section 3.1. A summary of this paper is finally presented in Section 4.

\section{Power law distribution of high-flow states}

As high flows are unstable to medium-sized perturbations $[3,5]$, it is important to learn more about their statistics. Here, we define "high-flow states" by exceeding a given flow threshold $Q_{\text {thr }}$ (which may be chosen much smaller than the maximum flow $Q_{\max }$ or the dynamic capacity $Q_{\text {out }}$, i.e. the outflow from seriously congested traffic $)^{2}$.

In the following, we study the duration of "high flow states" by analyzing single-vehicle data of the Dutch freeway A9 from Haarlem to Amsterdam in the Netherlands (see Refs. $[9,16,41]$ for details of the data). Specifically, we aggregated the data to obtain 2 -min averages $Q(x, t)$ of the flow as a function of time $t$ at a certain location $x$ of the freeway. Afterwards, we determined the frequency of time periods $\Delta T$ over which the flows $Q(x, t)$ stayed above a certain threshold $Q_{\mathrm{thr}}$ (see Fig. 3). A representative example is shown in Figure 4.

Similar pictures as for $Q_{\mathrm{thr}}=1400$ vehicles $/ \mathrm{h}$ are found for other threshold values $Q_{\mathrm{thr}}$, but the data tend to be more noisy for large values of $Q_{\mathrm{thr}}$, as the typical durations of high-flow states become shorter. Generally,

\footnotetext{
$2 Q_{\text {out }}$ is typically of the order of 1800 vehicles per kilometer and lane. In contrast, $Q_{\max }$ in the left (fast) lane reaches values upto $Q_{\max }=3200$ vehicles per hour. In the right (truck) lane, the maximum flow does not exceed values of 2500 vehicles per hour. These values are for averages over 50 vehicles [16]. For further details about the studied data see references $[9,16]$.
} 


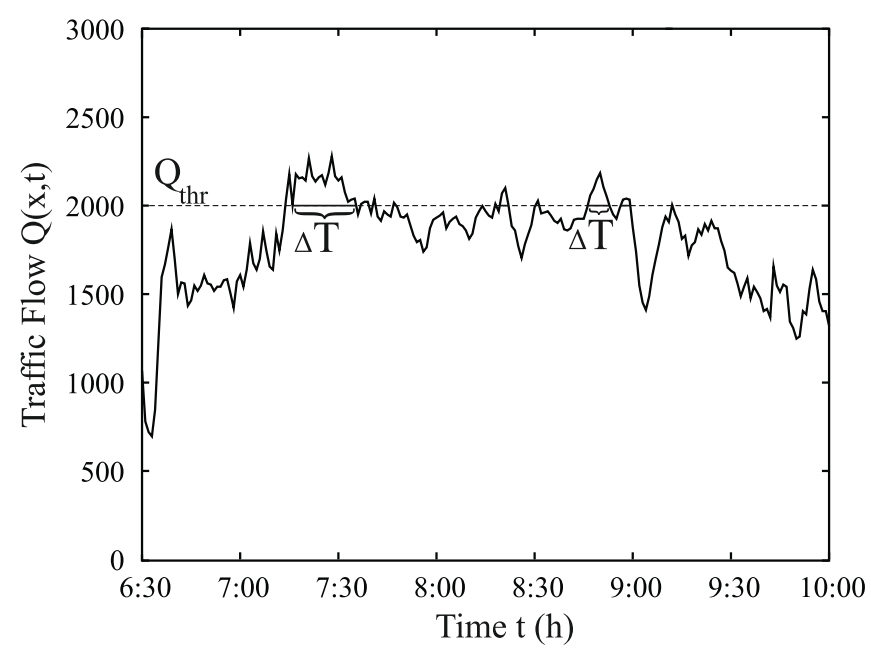

Fig. 3. Illustration of how the duration $\Delta T$ of high-flow states is determined. A given flow threshold $Q_{\text {thr }}$ cuts the timedependent curve $Q(x, t)$ of measured flows several time. The duration between two subsequent cutting points corresponds to a measurement of $\Delta T$, if the $Q(x, t) \geq Q_{\text {thr }}$ holds during this time period. These time periods are indicated by solid horizontal lines, while a dotted line was used if $Q(x, t)<Q_{\mathrm{thr}}$.

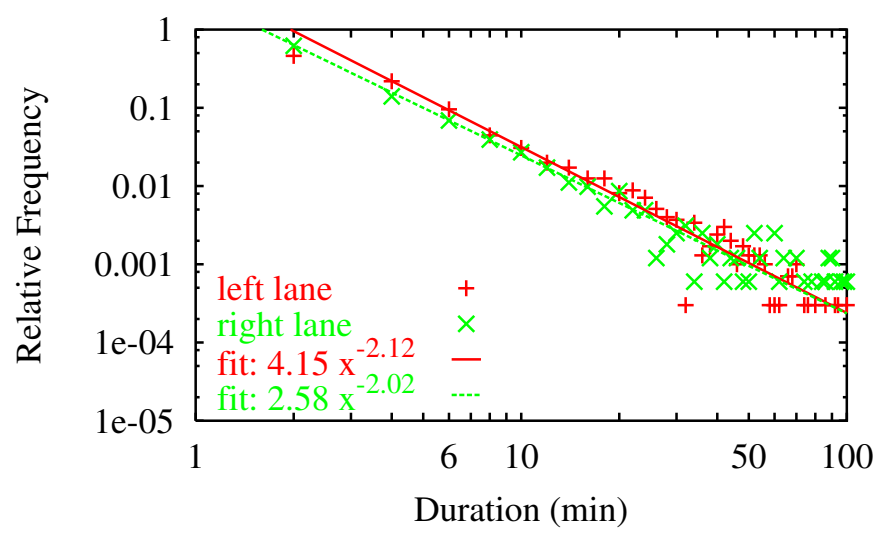

Fig. 4. Typical scaling law for the distribution of durations, for which the vehicle flow $Q(x, t)$ at the cross section $x$ of a freeway exceeds the threshold $Q_{\mathrm{thr}}=1400$ vehicles $/ \mathrm{h}$. The distribution follows a power law and has been determined from two-minute averages of single vehicle data of the Dutch freeway A9 from Rottepolderplein to Badheuvedorp close to Amsterdam.

however, the probability distribution $P(\Delta T)$ of durations of high-flow states can be surprisingly well approximated by the power law

$$
P(\Delta T)=F_{\mathrm{thr}} \Delta T^{-\alpha},
$$

where $F_{\text {thr }}$ is a proportionality factor and $\alpha$ the power law exponent. As the distribution $P(\Delta T)$ obviously depends on the chosen threshold value $Q_{\mathrm{thr}}$, it would be natural to assume that not only the proportionality factor $F_{\text {thr }}$, but also the respective power law exponent $\alpha$ depends on $Q_{\mathrm{thr}}$. However, Figure 5 suggests that we have $\alpha \approx 2$, irrespective of the value of $Q_{\mathrm{thr}}$. Therefore, we need to

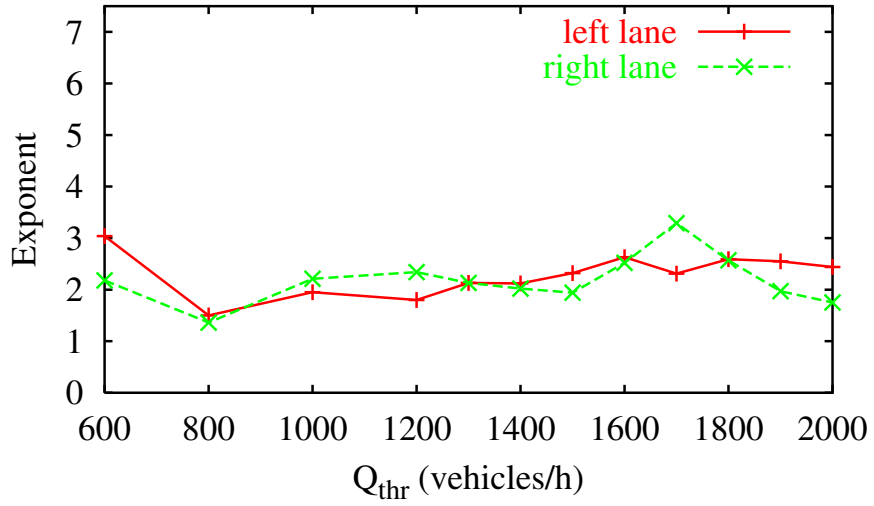

Fig. 5. Exponents of the power law of high-flow states, see equation (2), for different flow thresholds $Q_{\mathrm{thr}}$. One can see that the exponents, fitted within the interval $\Delta T \in[6,50] \mathrm{min}$, are of the order of 2 in both lanes. The exponents have been determined from two-minute averages of single vehicle data of the Dutch freeway A9 close to Amsterdam (after [43]).

find an explanation for both, the occurence of the power law and the value $\alpha \approx 2$ of its exponent. Note, however, that the results of Figure 5 are for a two-lane freeway with different speed limits for cars and trucks. In fact, for the three-lane freeway A8 close to Munich, Germany, there is empirical evidence of power laws, but the exponents somewhat depend on the freeway lane [42]. This will be relevant for the interpretation of the power law.

\section{Interpretation of the power law}

\subsection{Explanation of $1 / f$ fluctuations by single-lane models}

The empirical power law is quite intriguing, and its interpretation appears to be far from obvious. As the flow is basically given by the inverse time gaps, however, periods of high flow must be characterized by short time gaps between vehicles. Therefore, rather than a homogeneous flow with uniform time gaps between vehicles, the explanation of the above power law (2) must be related to the occurence of vehicle clusters. Their statistics must be characterized by largely varying sizes, to generate a time-dependence of the flow which shares features with a fractal curve $[44,46]$.

It is conceivable that such a dynamics could be produced by single-lane traffic models implementing a complex vehicle dynamics. In fact, previous publications have revealed $1 / f^{\alpha}$ fluctuations of the flow and other quantities in empirical data [45] and various traffic models. This includes cellular automata models [46-51], coupled maps [52], and fluid-dynamics traffic models [45,53]. As none of the publications has studied the frequency distribution of high-flow states we are focusing on in this paper, it is hard to say whether it is reproduced by any of these models. However, since the respective power law 
exponents depend on the choice of the model, it is conceivable that equation (2) could be reproduced by suitable specifications of one-lane models.

A comparison with the models discussed in Section 1 shows that, besides stochasticity, such a model would have to contain non-linear vehicle interactions. Otherwise all cars would finally queue up behind the slowest vehicle(s) with identical time gaps, which does not reproduce the empirical data. $1 / f$ fluctuations of traffic flows can, for example, be explained by turbulent behavior of the noisy Burgers equation [45]. They can also be derived as result of self-organized criticality, if the traffic state with the largest throughput is critical and characterized by jams of all sizes $[46,47]$. As the dynamics of the underlying particle hopping models can be related to the stochastic Burgers equation [54], both approaches are mutually consistent. However, both of them are not so well compatible with some empirically observed features of congested traffic flows $[55,56]$. For this reason, the above traffic models have been continuously refined.

In recent models, noise is much less dominating as compared to the interactions between vehicles, and it is therefore questionable whether these models show $1 / f$ fluctuations in the flow dynamics or the power-law (2) of high-flow states. Furthermore, data for a three-lane freeway show power laws, but their exponents depend on the flow threshold $Q_{\text {thr }}$ and somewhat on the lane. In particular, they can be different from 2. Therefore, modeling the high-flow power law based on longitudinal vehicle dynamics may not be the best possible approach, as it cannot explain the difference between a freeway with 2 or more lanes. We rather think that one needs to take into account the interactions between lanes, i.e. the transversal dynamics. The model suggested in the following is a first modeling attempt in this direction.

While the above one-lane models basically assume one vehicle type and find $1 / f$ fluctuations primarily in the congested regime, the following proposal assumes two distinctly different vehicle types (cars and trucks) and implies a high-flow power law already before the traffic flow breaks down (i.e. before traffic becomes unstable and a capacity drop occurs).

\subsection{Multi-lane model of high-flow states based on vehicle bunching behind overtaking cars}

While we do not exclude the possibility of other modeling approaches, we will now focus on an interpretation of our empirical observations, assuming heterogeneous traffic including overtaking maneuvers. As we will see, this predicts a power law for high-flow states even for deterministic models of longitudinal vehicle interactions. Our analysis focuses on the case of a two-lane freeway, and it contains several steps:

1. first we will give a qualitative analysis of lane changing processes;

2. we will argue that they can cause the formation of vehicle platoons, and that this process is dominated by overtaking maneuvers at low speed, i.e. primarily by overtaking maneuvers of trucks;

3. we will determine the statistics of the duration of overtaking maneuvers by trucks from their speed distribution;

4. we will give reasons why they couple the dynamics on both freeway lanes and why the platoons forming on both lanes are expected to be comparable in size;

5 . we will analyze the length of forming vehicle platoons and the time period until they dissolve;

6. we will show that high-flow vehicle clusters persist for a very long time, even after the dissolution of the vehicle platoons:

7. we will discuss, what will be the result, if such vehicle clusters are measured at a local cross section of the freeway.

It will turn out that both, the lifetime of vehicle platoons and the local measurement of vehicle clusters scale with the duration of the overtaking maneuvers of trucks, and that the power law of high-flow states results as a superposition of power laws with the same exponent $\alpha=2$.

\subsubsection{Qualitative analysis of overtaking maneuvers on two-lane freeways}

In the following, we will assume that the desired velocities of vehicles are heterogeneous (see Fig. 6). This is typical for many European countries including Germany and the Netherlands, where the use of automatic cruise control is not common. The heterogeneity of desired speeds typically implies relative velocities among neighboring cars. The faster car will usually try to overtake a slower vehicle in front. We can distinguish two situations: either the traffic situation on the lane to the left will admit an overtaking maneuver ${ }^{3}$, or the vehicle will be obstructed by a slower vehicle and decelerate to its speed. During light traffic conditions, we may assume unobstructed overtaking most of the time, but already at moderate densities, long before the breakdown of traffic flow, obstructions occur. These obstructions persist for a certain time period $\Delta t$ and lead to the formation of platoons of cars.

Besides the duration of the overtaking maneuver, the size of the forming vehicle platoon depends primarily on two factors: (1) the absolute speed difference between the overtaking vehicle and the ones following it and (2) the surrounding vehicle density $\rho_{\mathrm{fr}}$. As our high flow power law concerns time periods from 2 min to $2 \mathrm{~h}$, we are particularly interested in large vehicle clusters and, therefore, in the overtaking maneuvers of trucks. These are characterized by particularly long durations $\Delta t$ and by a large absolute speed difference with respect to following cars, so that a considerable number of vehicles can accumulate behind the overtaking trucks.

On a two-lane freeway, the overtaking maneuver of trucks will create a moving bottleneck [57] on both lanes, while on a freeway with three or more lanes, passing of

\footnotetext{
${ }^{3}$ In Great Britain or Japan, for example, it will be the lane
} to the right. 


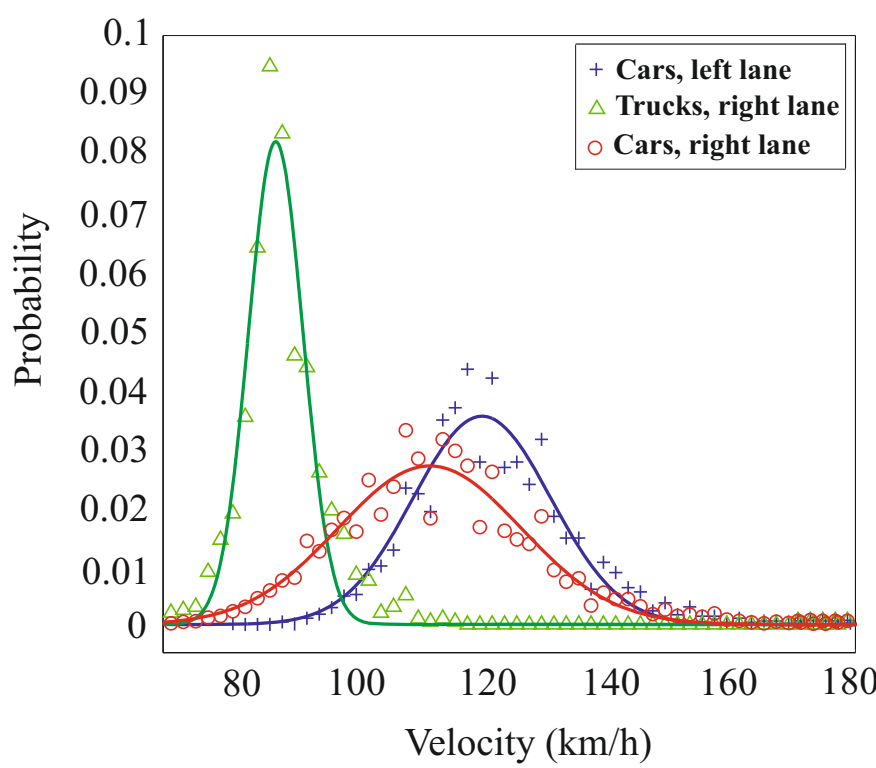

Fig. 6. Speed distributions of cars and trucks under free traffic conditions. Normal distributions fit the data reasonably well, in agreement with theoretical results. The distributions have been determined from single vehicle data of the Dutch freeway A9 close to Amsterdam. Note that the left lane is the fast lane and that the speed limits for cars is $120 \mathrm{~km} / \mathrm{h}$ on this freeway stretch, while it is $80 \mathrm{~km} / \mathrm{h}$ for trucks.

vehicles may still be possible ${ }^{4}$. The moving bottleneck propagates at the speed of trucks, i.e. at $V_{\mathrm{tr}} \approx 80 \mathrm{~km} / \mathrm{h}$. Behind this moving bottleneck, more and more vehicles will accumulate, which generates growing vehicle platoons (see Fig. 7). Due to lane-changing maneuvers of impatient drivers, the vehicle platoons on both lanes are expected to grow approximately at the same speed. Such synchronized behavior of neighboring lanes under obstructed flow conditions is well-known [59]. However, our calculations below would also work, if the platoon in one lane would grow faster by a certain proportionality factor.

\subsection{Duration of overtaking maneuvers of trucks}

Figure 6 shows the speed distributions of cars and trucks in free traffic of low vehicle density. It can be seen that the speed of trucks varies around the applicable speed limit for trucks of $80 \mathrm{~km} / \mathrm{h}$, and that the distribution is quite narrow (i.e. speed differences are small). Furthermore, the speed distributions of cars and trucks can be reasonably well approximated by Gaussian distributions [61,62], in agreement with theoretical predictions $[63,64]$.

Note that there is no distribution of desired speeds for trucks in the left lane, as they have to stay in the right lane according to applicable traffic law (when they do not overtake other vehicles). Overtaking maneuvers are started whenever two trucks in closeby locations move at

\footnotetext{
${ }^{4}$ How this can account for the different exponents observed in reference [42] shall be studied in another paper.
}

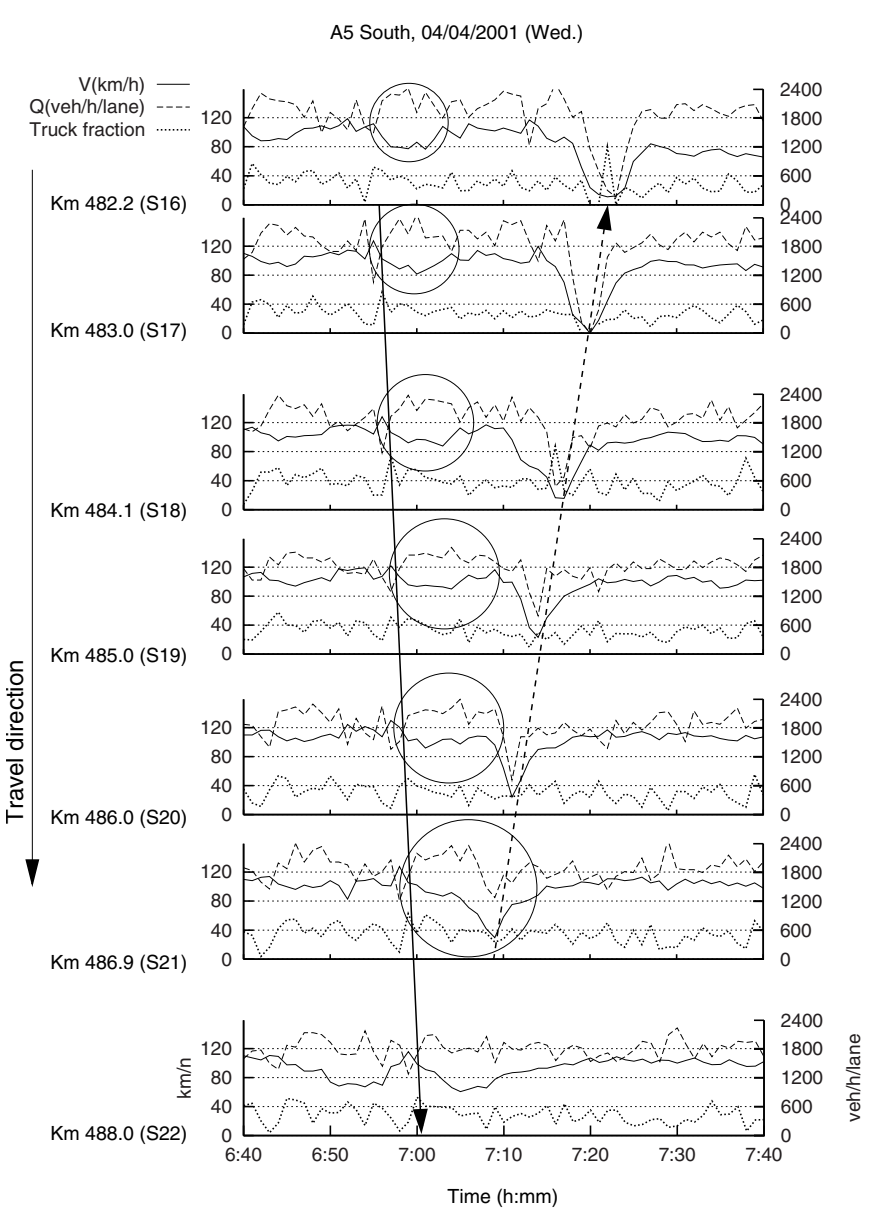

Fig. 7. Illustration of a traffic breakdown detected on the German freeway A5 close to Frankfurt (after [56]). The measurement of a peak in the truck fraction (see solid arrow) is followed by a time period of lower speeds and higher flows (see circles). The spatial extension of the high-flow area is growing (symbolized by larger circles), while it propagates along the freeway with about $75 \mathrm{~km} / \mathrm{h}$, i.e. at about the truck speed. We interpret this as growing vehicle platoons behind overtaking trucks. Approximately at kilometer 487, shortly upstream of a freeway junction characterized by many disturbing lane changes, the perturbation in the traffic flow starts to travel upstream. While we had a growing spatial extension before, we now find a growing perturbation amplitude (see dashed arrow). This eventually causes a breakdown of the traffic flow.

different speeds, no matter how small the relative velocity is ${ }^{5}$. Trucks often enter the left lane relentlessly, without waiting for a gap, i.e. vehicles in the left lane basically have to break if a truck driver decides to move over to the left lane. Moreover, during the overtaking maneuver, the speed of the trucks usually stays the same ${ }^{6}$. Therefore, the distribution of speed differences $\Delta v$ between an overtaking and an overtaken truck can be derived from the distribution of desired truck speeds in the right lane.

\footnotetext{
5 Therefore, their frequency depends on the truck density.

6 Trucks, particularly when fully loaded, can hardly accelerate and avoid decelerating.
} 
From statistics, it is well-known that the difference of two identically, independently, Gaussian distributed variables is Gaussian distributed as well. That is, the relative speeds $\Delta v$ of trucks obey a Gaussian distribution

$$
N(\Delta v) d \Delta v=\frac{1}{\sqrt{2 \pi \theta}} e^{-(\Delta v)^{2} /(2 \theta)} d \Delta v .
$$

This will be relevant for the derivation of the high-flow power law later on.

We will now determine the distribution of the time period $\Delta t$ required for the overtaking maneuver of trucks. If $\Delta l_{\text {eff }}$ denotes the typical effective distance, over which an overtaking maneuver takes place (e.g. $\Delta l_{\text {eff }}=20 \mathrm{~m}$ ) and $\Delta v$ is the relative speed between an overtaking vehicle and the slower one it overtakes, we find the proportionality relation

$$
\Delta t=\frac{\Delta l_{\mathrm{eff}}}{\Delta v} .
$$

As a consequence, the distribution of $\Delta t$ is given by the distribution of $1 / \Delta v$.

We will now derive the distribution of the variable $y=$ $1 / \Delta v$ from equation (3) by an appropriate transformation. Considering

$$
\frac{d y}{d \Delta v}=-\frac{1}{\Delta v^{2}}=-y^{2}
$$

we find

$$
N(\Delta v) d \Delta v=-N(\Delta v) \frac{1}{y^{2}} d y
$$

where the minus sign is compensated for by integration from small to large values of $y=1 / \Delta v$ rather than vice versa. Therefore, we get the distribution

$$
P(y) d y=\frac{1}{\sqrt{2 \pi \theta}} \frac{1}{y^{2}} e^{-1 /\left(2 \theta y^{2}\right)} d y .
$$

In the limit of small speed differences $\Delta v$, i.e. large values of $y$, we finally obtain the power law

$$
P(y) d y \sim y^{-2} d y
$$

Furthermore, as $y=1 / \Delta v \sim \Delta t$, see equation (4), this implies the power-law distribution

$$
P(\Delta t) d \Delta t \sim(\Delta t)^{-2} d \Delta t .
$$

As this power law results in the limit of small $\Delta v$, equation (9) is also found, if the distribution of speed differences of trucks is approximately Gaussian. With this, we mean

$$
N(\Delta v) d \Delta v=\mathcal{N} e^{-p(\Delta v)} d \Delta v
$$

with a normalization constant $\mathcal{N}$ and a Taylor expansion

$$
p(\Delta v)=a_{2}(\Delta v)^{2}+a_{3}(\Delta v)^{3}+a_{4}(\Delta v)^{4}+\ldots
$$

Hence, our conclusions would stay the same, if the distribution would be approximately Gaussian around $\Delta v=0$. This should be true under the assumptions made above, namely that an overtaking truck does not change its speed. According to the observations of the authors, this assumption appears to be quite realistic.
We have now to study, how the distribution of time periods $\Delta t$ translates into vehicle platoons behind the trucks and into high-flow states measured at local cross sections of the freeway. In particular, we have to explain why the distribution of high-flow states extends up to $100 \mathrm{~min}$, while overtaking maneuvers of trucks only take a few minutes (in the worst case).

\subsubsection{Platoon formation during overtaking maneuvers}

As discussed above, it is natural that overtaking maneuvers of slow vehicles, particularly trucks, constitute moving bottlenecks for faster vehicles for some time. These faster vehicles will be queued up behind the slow ones (the trucks) and form a platoon. We will now determine the growth of the platoon length. Intuitively, the number of vehicles in the platoon grows proportionally with the time $\Delta t$ required for the overtaking maneuver of the slow vehicles.

To quantify this, we may apply the well-known formula for shock propagation (i.e. the propagation of fronts between areas of different density) [65]. Let $V_{\mathrm{e}}(\rho)$ be the equilibrium speed-density relation and $\rho_{\text {fr }}$ the density per lane upstream of the moving bottleneck and the forming vehicle platoon. Moreover, let $\rho_{\mathrm{pl}}>\rho_{\mathrm{fr}}$ be the density per lane corresponding to the speed $V_{\mathrm{tr}} \approx 80 \mathrm{~km} / \mathrm{h}$ of trucks, i.e.

$$
V_{\mathrm{e}}\left(\rho_{\mathrm{pl}}\right)=V_{\mathrm{tr}}
$$

Finally, let

$$
Q_{\mathrm{e}}(\rho)=\rho V_{\mathrm{e}}(\rho)
$$

represent the equilibrium flow-density relation, which is sometimes called the "fundamental diagram" 7 . Then, the propagation speed of the upstream end of the vehicle platoon forming behind the moving bottleneck is expected to be

$$
C\left(\rho_{\mathrm{fr}}\right)=\frac{Q_{\mathrm{e}}\left(\rho_{\mathrm{pl}}\right)-Q_{\mathrm{e}}\left(\rho_{\mathrm{fr}}\right)}{\rho_{\mathrm{pl}}-\rho_{\mathrm{fr}}}=\frac{\rho_{\mathrm{pl}} V_{\mathrm{tr}}-\rho_{\mathrm{fr}} V_{\mathrm{e}}\left(\rho_{\mathrm{fr}}\right)}{\rho_{\mathrm{pl}}-\rho_{\mathrm{fr}}} .
$$

Relative to the truck speed $V_{\mathrm{tr}}$, the propagation speed of the upstream platoon front can be calculated as

$$
c\left(\rho_{\mathrm{fr}}\right)=C\left(\rho_{\mathrm{fr}}\right)-V_{\mathrm{tr}}=-\frac{V_{\mathrm{e}}\left(\rho_{\mathrm{fr}}\right)-V_{\mathrm{tr}}}{\rho_{\mathrm{pl}} / \rho_{\mathrm{fr}}-1}<0 .
$$

The negative values imply that the platoon is growing backwards, as expected. Therefore, the platoon length $l_{\mathrm{pl}}(t)$ grows linearly with the time $t$ at the rate $\left|c\left(\rho_{\mathrm{fr}}\right)\right|$. After an obstructed time period of duration $\Delta t$, the platoon is expected to have the length

$$
l_{\mathrm{pl}}(\Delta t)=|c| \Delta t .
$$

Note that, in this paper, we assume that the vehicle density $\rho_{\mathrm{pl}}$ in the platoon is (meta)stable, i.e.

$$
\rho_{\mathrm{pl}}<\rho_{\mathrm{c} 2},
$$

7 While the flow-density data scatter largely in the congested area, here we require the flow-density relationship in the density regime before the capacity drop, which is well defined. 
see references [6,58]. This situation may be compared with a "widening synchronized pattern" $[58,60]$, but with a moving rather than stationary downstream front. The density $\rho_{\mathrm{c} 2}$ represents the threshold to linearly unstable traffic.

If relationship (17) is given, traffic flow only destabilizes and suffers a breakdown of capacity ${ }^{8}$, if there are merging flows or strong local perturbations (see the example in Fig. 7). Without such perturbations, the platoons will expand in space without a breakdown of the flow. Therefore, the outflow from the vehicle clusters, after the trucks have finished their overtaking maneuver, is given by the free flow capacity $Q_{\max }$ rather than the congested flow capacity $Q_{\text {out }}[58]$.

\subsubsection{Dissolution of vehicle platoons}

After the overtaking maneuver is completed and the related obstruction of the vehicle flow has ended, the vehicle platoon dissolves linearly in time. Let us denote the maximum flow by $Q_{\max }$ and the related density by $\rho_{\max }$, where $Q_{\mathrm{e}}\left(\rho_{\max }\right)=Q_{\max }$. According to the shock wave equation for the propagation of discontinuous density changes [65], the dissolution speed is then given by [58]

$$
C_{*}=\frac{Q_{\mathrm{e}}\left(\rho_{\max }\right) / 2-Q_{\mathrm{e}}\left(\rho_{\mathrm{pl}}\right)}{\rho_{\max }-\rho_{\mathrm{pl}}}=\frac{\rho_{\max } V_{\mathrm{e}}\left(\rho_{\max }\right) / 2-\rho_{\mathrm{pl}} V_{\mathrm{tr}}}{\rho_{\max }-\rho_{\mathrm{pl}}},
$$

where the factor $1 / 2$ takes into account that only one of the two lanes, namely the left lane is free to dissolve the vehicle platoon, after the overtaking maneuver of trucks is finished. Relative to the truck velocity $V_{\mathrm{tr}}$, the propagation speed is

$$
c_{*}=C_{*}-V_{\mathrm{tr}}=-\frac{V_{\mathrm{e}}\left(\rho_{\max }\right) / 2-V_{\mathrm{tr}}}{\rho_{\mathrm{pl}} / \rho_{\max }-1} .
$$

The dissolution process takes a time period $\Delta t_{*}$. While the vehicle platoon dissolves at the front, further vehicles are joining it at its end. Therefore, we have the relation

$$
l_{\mathrm{pl}}(\Delta t)+|c| \Delta t_{*}=\left|c_{*}\right| \Delta t_{*} .
$$

This results in

$$
\Delta t_{*}=\frac{|c| \Delta t}{\left|c_{*}\right|-|c|}=\frac{\Delta t}{\left|c_{*}\right| /|c|-1} .
$$

Hence, the existence of a platoon is observed for an overall time period of

$$
\Delta T_{*}=\Delta t_{*}+\Delta t=\frac{\Delta t}{1-|c| /\left|c_{*}\right|} \geq \Delta t .
$$

The quotient in the denominator can be determined as

$$
\frac{|c|}{\left|c_{*}\right|}=\frac{\rho_{\mathrm{fr}}}{\rho_{\max }} \frac{\left|\frac{V_{\mathrm{e}}\left(\rho_{\mathrm{fr}}\right)-V_{\mathrm{tr}}}{\rho_{\mathrm{pl}}-\rho_{\mathrm{fr}}}\right|}{\frac{V_{\mathrm{e}}\left(\rho_{\max }\right) / 2-V_{\mathrm{tr}}}{\rho_{\mathrm{pl}}-\rho_{\max }} \mid} .
$$

\footnotetext{
${ }^{8}$ Namely, from the maximum flow $Q_{\max }$ to the outflow $Q_{\text {out }}$ from seriously congested traffic.
}

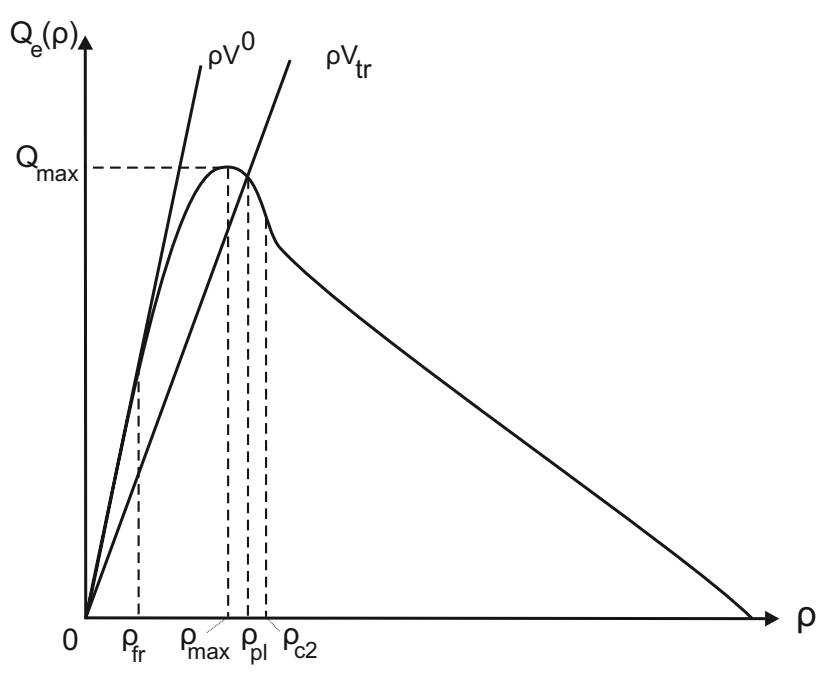

Fig. 8. Illustration of the various densities and flows determining the formation and dissolution of vehicle platoons. We have $\rho_{\text {fr }} \leq \rho_{\max }$ and assume $\rho_{\max }<\rho_{\mathrm{pl}}<\rho_{\mathrm{c} 2}$, which supports the (meta-)stability of vehicle platoons. Therefore, they may persist over a considerable time period without a breakdown of "high-flow states".

Note the relationships

$$
\rho_{\mathrm{fr}} \leq \rho_{\max }<\rho_{\mathrm{pl}}
$$

and

$$
V_{\mathrm{e}}\left(\rho_{\mathrm{fr}}\right) \geq V_{\mathrm{e}}\left(\rho_{\max }\right)>V_{\mathrm{e}}\left(\rho_{\mathrm{pl}}\right)
$$

(see Fig. 8). Therefore, equations (22) and (23) imply a platoon to exist for a time period $\Delta T_{*}=\Delta t$, if $\rho_{\mathrm{fr}} \rightarrow 0$, while it will exist for an extremely long time as $Q_{\mathrm{e}}\left(\rho_{\mathrm{fr}}\right) \rightarrow$ $Q_{\max } / 2$. Consequently, at low vehicle densities, most platoons are expected to dissolve quickly. However, at moderate densities, the lifetime $\Delta T_{*}=\Delta t+\Delta t_{*}$ can be quite long and even diverge. This (and the persistence of highflow vehicle clusters after the dissolution of platoons, see next subsection) is the reason, why overtaking maneuvers on the scale of minutes can have effects on much longer time scales.

\subsubsection{Measurement of high-flow states}

We will now have to relate the existence of vehicle platoons to the local measurement of high-flow states. When a vehicle platoon passes a cross section of the road, the traffic flow is particularly high due to the small time gaps between its vehicles. In comparison with the situation before the overtaking maneuver of trucks, the time gaps between the freely flowing cars are considerably reduced. That is, the flow is still higher than $Q_{\mathrm{e}}\left(\rho_{\mathrm{fr}}\right)$, the flow before platoon formation, even after the vehicle platoon has dissolved. The related vehicle clusters are expected to exist for a long time, much longer than the previous vehicle platoon $^{9}$. The corresponding flows in both lanes qualify as high-flow states, if they are larger than $Q_{\mathrm{thr}}$.

9 There are basically two ways for them to disappear: 1) vehicles leave the cluster via off-ramps of the freeway; 2) clusters 
Let us now estimate the flow in both lanes after the overtaking maneuver of trucks is finished. Then, vehicles will pass the truck in the right lane at the maximum flow rate $Q_{\max }$. This flow will be distributed over both freeway lanes. A fraction $\beta=\beta\left(\rho_{\mathrm{fr}}\right)$ will switch to the right lane, while a fraction $(1-\beta)$ will stay on the left lane. In the right lane, in front of the overtaken truck, there will be some additional truck traffic. If $\gamma$ denotes the average fraction of truck traffic on both freeway lanes, this corresponds to an average truck traffic flow of $Q_{\mathrm{tr}} \approx \gamma Q_{\mathrm{e}}\left(\rho_{\mathrm{fr}}\right)$. Considering that most of these trucks are in the right lane, the overall flow on the right lane after a completed overtaking maneuver of trucks may be estimated as

$$
Q_{\text {right }}\left(\rho_{\mathrm{fr}}, t\right) \approx \beta\left(\rho_{\mathrm{fr}}\right) Q_{\max }+2 \gamma(t) Q_{\mathrm{e}}\left(\rho_{\mathrm{fr}}\right) .
$$

In the left lane, we approximately expect to find the flow

$$
Q_{\text {left }}\left(\rho_{\mathrm{fr}}, t\right) \approx\left[1-\beta\left(\rho_{\mathrm{fr}}\right)\right] Q_{\max } .
$$

While the flow $Q_{\text {left }}$ is typically above 2000 vehicles per hour (we estimate that $\beta \approx 0.35$ ), the flow in the right lane exceeds high flow thresholds $Q_{\text {thr }}$ as well, if the surrounding density $\rho_{\text {fr }}$ and the truck fraction $\gamma$ are large enough. Therefore, after an overtaking maneuver of trucks, we expect to find high-flow states in both lanes ${ }^{10}$. We will now have to determine the time period over which they are measured.

Previously, we have studied vehicle clusters in a moving coordinate system, specifically in a system moving at the speed of trucks. Our empirical distribution of high-flow states, however, has been measured at a local cross section of a two-lane freeway. In order to see how this affects the measurement, it is useful to consult Figure 9.

We see that the time period $\Delta T$ of high-flow clusters is basically given by $\Delta t_{*}$, which is proportional to $\Delta t$, see equation (21). The proportionality factor

$$
A\left(\rho_{\mathrm{fr}}\right)=\frac{1}{\left|c_{*}\right| /\left|c\left(\rho_{\mathrm{fr}}\right)\right|-1}
$$

depends on the surrounding vehicle density $\rho_{\mathrm{fr}}$. But given a certain density $\rho_{\text {fr }}$, we have the proportionality $\Delta T^{-2} \sim$ $\Delta t^{-2}$. Together with the power law distribution (9) for $\Delta t$, this implies the power law distribution

$$
P\left(\Delta T, \rho_{\mathrm{fr}}\right) \sim(\Delta T)^{-2} \sim(\Delta t)^{-2} .
$$

As only the proportionality factor, but not the exponent $\alpha=2$ depends on the density $\rho_{\mathrm{fr}}$, the superposition of distributions for different values of $\rho_{\mathrm{fr}}$ is again a power law with exponent $\alpha=2$ :

$$
P(\Delta T) \sim(\Delta T)^{-2} \sim \Delta t^{-2} .
$$

Therefore, we have shown that the measured duration $\Delta T$ of vehicle platoons is distributed according to a power law

disperse due to a variation of the vehicle speeds, which can be approximated by continuity equations for cars and trucks with diffusion terms, see the appendix.

10 At least if $Q_{\text {left }}, Q_{\text {right }}>Q_{\text {thr }}$ and $Q_{\max }+2 \gamma Q_{\mathrm{e}}\left(\rho_{\text {fr }}\right)>$ $2 Q_{\mathrm{e}}\left(\rho_{\text {fr }}\right)$.

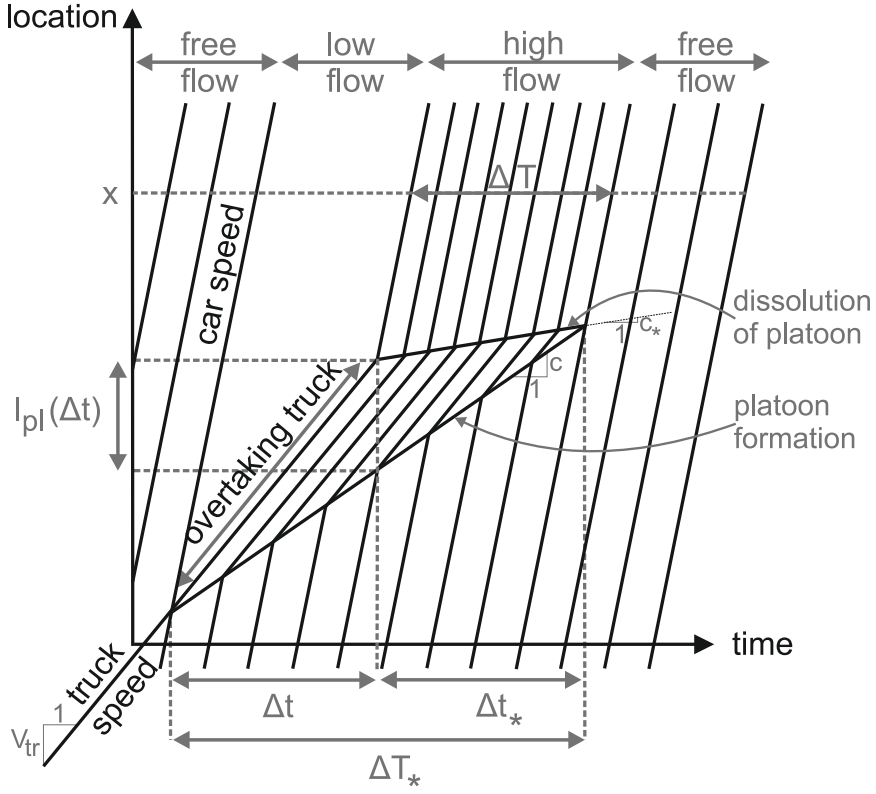

Fig. 9. Schematic illustration of vehicle trajectories on the left lane before, during, and after an overtaking maneuver of trucks. The triangular area represents the growth and dissolution of a vehicle cluster. When measuring the flow after the dissolution of the platoon at some freeway cross section $x$, one expects to detect the free flow $Q_{\mathrm{e}}\left(\rho_{\mathrm{fr}}\right)$, then a low-flow period in front of the overtaking trucks, afterwards a high-flow period, and finally again free flow. The measured duration $\Delta T$ of the high-flow state, which is expected to persist for a long time, is basically given by $\Delta t_{*}$.

$P(\Delta T) \sim(\Delta T)^{-\alpha}$ with $\alpha=2$, in agreement with the empirical measurement of high-flow states represented by equation (2).

\section{Summary and outlook}

In this paper, we have revealed a power law for the duration of high-flow states, where "high flow" means higher than some given threshold $Q_{\mathrm{thr}}$. Not only was it surprising to find that our empirical data could be approximated by a power law, but also that the power law exponents $\alpha$ for the lanes of a two-lane freeway were approximately 2 , irrespective of the flow threshold $Q_{\mathrm{thr}}$.

Therefore, it was natural to look for an explanation of these surprising findings. As the dependence of the exponents on the number of freeway lanes does not support explanations based on the longitudinal vehicle dynamics, we have studied a model based on the transversal vehicle dynamics, i.e. lane changes ${ }^{11}$. Our hypothesis was that high-flow states occured due to vehicle platoons, and that these vehicle platoons would be caused by lasting overtaking maneuvers of slow vehicles, particularly of trucks.

\footnotetext{
11 An alternative modeling approach, however, may be based on modifications of the one-lane models for vehicle platoons discussed in Section 1, if the exponent is (made) dependent on the number of lanes.
} 
Based on an approximate Gaussian distribution of the speed difference of trucks, we could, in fact, derive the empirical power law and the exponent $\alpha=2$. For this, we have studied the duration of overtaking maneuvers, the resulting lifetime of forming vehicle platoons, and the measurement process at a cross section of the freeway. The lifetime $\Delta T_{*}$ of a vehicle platoon and the measurement $\Delta T$ of the related high-flow vehicle cluster are proportional to the duration $\Delta t$ of overtaking maneuvers. This circumstance transfers the power law of the time periods $\Delta t$ to the periods of the high-flow states. The powerlaw itself results from the approximate Gaussian distribution of relative speeds $\Delta v$, the fact that the duration $\Delta t$ of overtaking maneuvers is inversely proportional to $\Delta v$, and the domination of long-lasting overtaking maneuvers with $\Delta v \approx 0$.

Author contributions: B.T. performed the empirical data analysis, while D.H. developed the theoretical model.

The authors would like to thank Rudolf Sollacher for stimulating the empirical investigation of the characteristics of highflow states and the Dutch Ministry of Transport, Public Works and Water Management, in particular Henk Taale, for providing the data of the Dutch freeway A9. The data of the German freeway A5 were provided by the Hessisches Landesamt für Straßen- und Verkehrswesen. Peter Felten and Mehdi Moussaid fitted the Gauss curves to the data of Figure 5. We also thank Peter Felten for preparing the illustrations of Figures 5 and 8 .

\section{Appendix A: Dispersion of vehicle clusters}

For each vehicle class $a$ (i.e. cars and trucks), the dispersion of vehicle clusters is described by the traffic pressure $P_{a}(x, t)=\rho_{a}(x, t) \theta_{a}(x, t)$, which is proportional to the vehicle density $\rho_{a}(x, t)$ of vehicle type $a$ and its velocity variance $\theta_{a}(x, t)$ as a function of location $x$ and time $t[66]$. In the limit of small adaptation times $\tau_{a}$ for the velocity relaxation, the average speed $V_{a}$ of vehicle class $a$ may be approximated by

$$
V_{a}(x, t)=V_{a}^{\mathrm{e}}\left(\sum_{b} \rho_{b}(x, t)\right)-\frac{\tau_{a}}{\rho_{a}} \frac{\partial P_{a}(x, t)}{\partial x},
$$

where $V_{a}^{\mathrm{e}}\left(\sum_{b} \rho_{b}(x, t)\right)$ is the equilibrium relationship between speed $V_{a}$ and density $\rho(x, t)=\sum_{b} \rho_{b}(x, t)$. Inserting equation (A.1) into the continuity equation for each vehicle class, the dispersion of vehicle clusters due to different speeds can be reflected by diffusion terms $D_{a b} \partial^{2} \rho_{b} / \partial x^{2}$ (for details of the derivation see Ref. [67]):

$$
\frac{\partial \rho_{a}(x, t)}{\partial t}+V_{a}^{\mathrm{e}}\left(\sum_{b} \rho_{b}(x, t)\right) \frac{\partial \rho_{a}}{\partial x} \approx \sum_{b} \frac{\partial}{\partial x}\left(D_{a b} \frac{\partial \rho_{b}}{\partial x}\right) .
$$

Herein, the diffusion parameters $D_{a b}$ are given by

$$
D_{a b}\left(\rho_{\mathrm{a}}, \rho_{\mathrm{b}}\right)=\tau_{a} \frac{\partial P_{a}\left(\rho_{\mathrm{a}}, \rho_{\mathrm{b}}\right)}{\partial \rho_{b}} .
$$

The diffusion terms on the right-hand side of equation (A.2) imply a spatial dispersion of clusters in the course of time.

\section{References}

1. B.S. Kerner, P. Konhäuser, Phys. Rev. E 50, 54 (1994)

2. D. Chowdhury, L. Santen, A. Schadschneider, Physics Reports 329, 199 (2000)

3. D. Helbing, Rev. Modern Phys. 73, 1067 (2001)

4. T. Nagatani, Reports on Progress in Physics 65, 1331 (2002)

5. B. Persaud, S. Yagar, R. Brownlee, Transpn. Res. Rec. 1634, 64 (1998)

6. D. Helbing, M. Moussaid, Eur. Phys. J. B, in press, see e-print http://arxiv .org/abs/0807.4006

7. Y. Sugiyama, M. Fukui, M. Kikuchi, K. Hasebe, A. Nakayama, K. Nishinari, S.-I. Tadaki, S. Yukawa, New J. Phys. 10, 033001 (2008)

8. B.S. Kerner, H. Rehborn, Phys. Rev. E 53, R4275 (1996)

9. M. Treiber, D. Helbing, J. Phys. A: Math. Gen. 32, L17 (1999)

10. K. Nishinari, M. Treiber, D. Helbing, Phys. Rev. E 68, $067101(2003)$

11. M. Treiber, A. Kesting, D. Helbing, Phys. Rev. E 74, 016123 (2006)

12. J. Treiterer, J.A. Myers, in Proceedings of the 6th International Symposium on Transportation and Traffic Theory, edited by D. Buckley (Reed, London, 1974), pp. 13-38.

13. B.S. Kerner, H. Rehborn, Phys. Rev. E 53, R1297 (1996)

14. S. Krauss, P. Wagner, C. Gawron, Phys. Rev. E 55, 5597 (1997)

15. M. Cassidy, R.L. Bertini, Transportation Research B 33, 25 (1999)

16. B. Tilch, D. Helbing, in Traffic and Granular Flow '99, edited by D. Helbing et al. (Springer, Berlin, 2000), pp. 333-338.

17. M.R. Evans, Europhys. Lett. 36, 13 (1996)

18. J. Krug, P.A. Ferrari, J. Phys. A: Math. Gen. 29, L465 (1996)

19. V. Karimipour, Europhys. Lett. 47, 304 (1999)

20. V. Karimipour, Europhys. Lett. 47, 501 (1999)

21. T. Seppäläinen, J. Krug, J. Stat. Phys. 95, 525 (1999)

22. I. Benjamini, P.A. Ferrari, C. Landim, Stoch. Process. Appl. 61, 181 (1996)

23. D. Helbing, B.A. Huberman, Nature 396, 738 (1998)

24. E. Ben-Naim, P.L. Krapivsky, S. Redner, Phys. Rev. E 50, 822 (1994)

25. E. Ben-Naim, P.L. Krapivsky, Phys. Rev. E 56, 6680 (1997)

26. E. Ben-Naim, P.L. Krapivsky, J. Phys. A: Math. Gen. 31, 8073 (1998)

27. E. Ben-Naim, P.L. Krapivsky, Phys. Rev. E 59, 88 (1999)

28. T. Nagatani, J. Phys. A: Math. Gen. 28, L119 (1995)

29. T. Nagatani, J. Phys. Soc. Jpn 65, 3386 (1996)

30. T. Nagatani, H. Emmerich, K. Nakanishi, Physica A 255, $158(1998)$

31. M. Fukui, Y. Ishibashi, J. Phys. Soc. Jpn 65, 1868 (1996)

32. T. Nagatani, Physica A 284, 405 (2000) 
33. D. Ktitarev, D. Chowdhury, D.E. Wolf, J. Phys. A: Math. Gen. 30, L221 (1997)

34. W. Knospe, L. Santen, A. Schadschneider, M. Schreckenberg, Physica A 265, 614 (1999)

35. L. Neubert, L. Santen, A. Schadschneider, M. Schreckenberg, Phys. Rev. E 60, 6480 (1999)

36. M.N. Islam, P.C. Consul, Transpn. Res. B 25, 365 (1991)

37. R. Mahnke, N. Pieret, Phys. Rev. E 56, 2666 (1997)

38. R. Mahnke, J. Kaupuzs, Phys. Rev. E 59, 117 (1999)

39. R. Kühne, R. Mahnke, I. Lubashevsky, J. Kaupuzs, Phys. Rev. E 65, 066125 (2002)

40. D. Helbing, R. Jiang, M. Treiber, Phys. Rev. E 72, 046130 (2005)

41. D. Helbing, Verkehrsdynamik (Springer, Berlin, 1997)

42. R. Sollacher, Dynamical properties of highway traffic, Internal report of the BMBF project SANDY, grant No. $13 \mathrm{~N} 7092 / 2$

43. B. Tilch, Modellierung und Simulation selbstgetriebener Vielteilchensysteme mit Anwendung auf den Straßenverkehr (University of Stuttgart, 2000), unpublished

44. M. Takayasu, H. Takayasu, Fractals 1, 860 (1993)

45. T. Musha, H. Higuchi, Jpn J. Appl. Phys. 15, 1271 (1976)

46. M. Paczuski, K. Nagel, in Traffic and Granular Flow, edited by D.E. Wolf, M. Schreckenberg, A. Bachem (World Scientific, Singapore, 1996)

47. K. Nagel, M. Paczuski, Phys. Rev. E 51, 2909 (1995)

48. T. Nagatani, Phys. Rev. E 51, 922 (1995)

49. X. Zhang, G. Hu, Phys. Rev. E 52, 4664 (1995)
50. D.V. Ktitarev, D. Chowdhury, D.E. Wolf, J. Phys. A: Math. Gen. 30, L221 (1997)

51. L. Roters, S. Lübeck, K.D. Usadel, Phys. Rev. E 59, 2672 (1999)

52. S. Yukawa, M. Kikuchi, J. Phys. Soc. Jpn 65, 916 (1996)

53. M.Y. Choi, H.Y. Lee, Phys. Rev. E 52, 5979 (1995)

54. K. Nagel, Phys. Rev. E 53, 4655 (1996)

55. B.S. Kerner, The Physics of Traffic (Springer, Berlin, 2004)

56. M. Schönhof, D. Helbing, Transportation Science 41, 135 (2007)

57. D.C. Gazis, R. Herman, Transpn. Sci. 26, 223 (1992)

58. D. Helbing, M. Treiber, A. Kesting, M. Schönhof, Eur. Phys. J. B, accepted for publication, see e-print http://arxiv.org/abs/0903.0929

59. B.S. Kerner, H. Rehborn, Phys. Rev. E 53, R4275 (1996)

60. B.S. Kerner, Transpn. Res. Rec. 1802, 145 (2002)

61. A.D. May, Traffic Flow Fundamentals (Prentice Hall, Englewood Cliffs, NJ, 1990)

62. M. Krbalek, D. Helbing, Physica A 333, 370 (2004)

63. M. Treiber, D. Helbing, Eur. Phys. J. B, submitted, see e-print http://arxiv.org/abs/0809.0426

64. C. Wagner, J. Stat. Phys. 90, 1251(1998)

65. M. Lighthill, G. Whitham, Proc. Roy. Soc. London A 229, $317(1955)$

66. D. Helbing, Eur. Phys. J. B, accepted for publication, see e-print http://arxiv.org/abs/0805.3400

67. D. Helbing, A. Greiner, Phys. Rev. E 55, 5498 (1997) 